\title{
A Digital Image Denoising Method with Edge Preservation Using Dyadic Lifting Schemes
}

\author{
Teruya Minamoto and Satoshi Fujii \\ Saga University, Saga, Japan \\ \{minamoto,fujii\}@ma.is.saga-u.ac.jp
}

\begin{abstract}
In this paper we proposed a new wavelet denoising method for digital images with edge preservation. Briefly stated, our method consists of a combination of the dyadic lifting schemes with the edgepreserving wavelet thresholding. The dyadic lifting schemes have free parameters which enable us to construct the filters having important image features. We describe how to determine these parameters and the denoising algorithm with edge preservation in detail. Some numerical experiments are presented, and we show that these parameters play an important role to denoise.
\end{abstract}

\section{Introduction}

Digital imaging has seen a huge progress over the last decade through the broad availability of digital cameras including web cameras and mobile phones. Since these digital images are often taken in poor conditions (e.g., photographing in a dark place), many images are corrupted by noise of several types. Therefore, the efficient image restoration methods are needed.

There are already commonly used spatial domain filters such as the weighted mean and median which can reduce corruption within images. In particular, PDEbased methods have been widely used for image denoising with edge preservation over the past few decades. These methods are either based on the nonlinear diffusions, or on the variational approach of energy functional minimization [1]. There are also an optimal Bayesian minimum mean square estimation (MMSE) based method to denoise 5. An alternative approach would be to use the frequency domains. Especially, wavelet transforms have been successfully employed in image processing. Donoho proposed the soft-threshold method in which the wavelet coefficients are below a certain threshold value reduced gradually to zero [2. This method based on down-sampling type of wavelet transform. However down-sampling wavelet is not shift-invariant, thus it tends to fail to carry out edge detection, feature extraction, and denoising. On the other hand, dyadic wavelet transform is shift-invariant as compared to discrete down-sampling type wavelet transform. Mallat has performed edge detection using discrete dyadic wavelet transform with quadratic spline dyadic wavelets [3.4].

In general, denoising algorithms have to balance the trade-off between denoising and preservation of structure. To overcome such a difficulty, there has

T. Wada, F. Huang, and S. Lin (Eds.): PSIVT 2009, LNCS 5414, pp. 283 294, 2009.

(C) Springer-Verlag Berlin Heidelberg 2009 
been a lot of work in the recent years on edge preserving algorithms. Standard algorithms are based on non-linear diffusion, or wavelets [18. Unfortunately, in spite of the sophistication of the recently proposed methods, most algorithms have not yet attained a desirable level of applicability. The study for efficient image denoising methods still is a valid challenge.

Very recently, the dyadic lifting schemes which extend Sweldens lifting schemes [7] is proposed in [6]. This dyadic lifting schemes have free parameters and two construction methods for custom-design of dyadic wavelets having desirable number of vanishing moments are also proposed in [6]. The authors designed spline dyadic wavelet filters with higher numbers of vanishing moments for denoising digital images and show its effectiveness in [6]. But the authors do not touch on the topic of edge-preserving, explicitly.

The purpose of this paper is to develop a new wavelet denoising method that exploit the dyadic lifting schemes and edge-preserving denoising method proposed in [8]. Differently from [6], we do not use their construction methods. We propose a new way to determine the free parameters in the dyadic lifting schemes which is suited to maintaining important image features instead.

This paper is organized as follows: in Section 2, we briefly introduce the dyadic lifting schemes which have the free parameters. The learning method to determine these free parameters is presented in Section 3. We describe the edge preserving method in Section 4 and the denoising algorithm in Section 5, Simulation results are demonstrated in Section 6, and Section 7 conclude this paper.

\section{Dyadic Lifting Schemes}

The arguments outlined in this section are very similar to those given in Refs. 346. We include this outline to make the present paper self-contained.

Let $L^{2}(\mathbb{R})$ be the space of square integrable functions on real line $\mathbb{R}$. We define the Fourier transform of the function $\psi \in L^{2}(\mathbb{R})$ by $\hat{\psi}(\omega)=\int_{-\infty}^{\infty} \psi(t) e^{-i \omega t} d t$. If there exist $A>0$ and $B$ such that

$$
A \leq \sum_{j=-\infty}^{\infty}\left|\hat{\psi}\left(2^{j} \omega\right)\right|^{2} \leq B
$$

then $\psi(t)$ is called dyadic wavelet function. It follows from (1) that $\hat{\psi}(0)=0$, i.e., $\int_{-\infty}^{\infty} \psi(t) d t=0$. Dyadic wavelet transform of $f(t)$ with the dyadic wavelet function $\psi(t)$ is defined by

$$
W f\left(u, 2^{j}\right)=\int_{-\infty}^{\infty} f(t) \frac{1}{\sqrt{2^{j}}} \psi\left(\frac{t-u}{2^{j}}\right) d t .
$$

To construct the dyadic wavelet function, we need a scaling function $\phi(t)$ satisfying a two-scale relation

$$
\phi(t)=\sum_{k} h[k] \sqrt{2} \phi(2 t-k) .
$$

The scaling function $\phi(t)$ is usually normalized as $\int_{-\infty}^{\infty} \phi(t) d t=1$. 
The Fourier transform of the scaling function (3) yields

$$
\hat{\phi}(\omega)=\frac{1}{\sqrt{2}} \hat{h}\left(\frac{\omega}{2}\right) \hat{\phi}\left(\frac{\omega}{2}\right),
$$

where $\hat{h}(\omega)$ denotes a discrete Fourier transform

$$
\hat{h}(\omega)=\sum_{k} h[k] e^{-i \omega k} .
$$

Since $\hat{\phi}(0)=1$ we can apply (4) and (5) to obtain $\hat{h}(0)=\sqrt{2}$ or $\sum_{k} h[k]=\sqrt{2}$. Using the scaling function $\phi(t)$ and the wavelet filter $g[k]$, a dyadic wavelet function is defined by $\psi(t)=\sum_{k} g[k] \sqrt{2} \phi(2 t-k)$. The expansion of any $f \in$ $L^{2}(\mathbb{R})$ by dyadic wavelet basis can be reconstructed under the reconstruction condition which is described in [4. To derive the reconstruction condition, a dual scaling function and a dual wavelet function are required. The dual scaling function $\tilde{\phi}(t)$ is defined by $\tilde{\phi}(t)=\sum_{k} \tilde{h}[k] \sqrt{2} \tilde{\phi}(2 t-k)$, and the dual wavelet function $\tilde{\psi}(t)$ is given by $\tilde{\psi}(t)=\sum_{k} \tilde{g}[k] \sqrt{2}(2 t-k)$.

Let us denote the discrete Fourier transforms of the filters $h[k], g[k], \tilde{h}[k], \tilde{g}[k]$ by $\hat{h}(\omega), \hat{g}(\omega), \hat{\tilde{h}}(\omega), \hat{\tilde{g}}(\omega)$, respectively. Then the reconstruction condition are described as

$$
\hat{\tilde{h}}(\omega) \hat{h}^{*}(\omega)+\hat{\tilde{g}}(\omega) \tilde{g}^{*}(\omega)=2, \quad \omega \in[-\pi, \pi],
$$

where the symbol $*$ denotes complex conjugation. The reconstruction condition (6) plays an important role in constructing lifting dyadic wavelet filters.

Proposition 1 (Ref.[6]). Suppose the discrete Fourier transforms $\hat{h}^{o}(\omega)$, $\hat{g}^{o}(\omega), \hat{\tilde{h}}^{o}(\omega)$, and $\tilde{\hat{g}}^{o}(\omega)$ of the initial filters $h^{o}[k], g^{o}[k], \tilde{h}^{o}[k]$ and $\tilde{g}^{o}[k]$, respectively, satisfy the reconstruction condition (6). Then, the Fourier transforms $\hat{h}(\omega), \hat{g}(\omega), \hat{\tilde{h}}(\omega)$, and $\tilde{\hat{g}}(\omega)$ of dual lifting dyadic wavelet filters defined by

$$
\begin{aligned}
h[k] & =h^{o}[k], \\
\tilde{h}[k] & =\tilde{h}^{o}[k]+\sum_{m} s[-m] \tilde{g}^{o}[k-m], \\
g[k] & =g^{o}[k]-\sum_{m} s[m] h^{o}[k-m], \\
\tilde{g}[k] & =\tilde{g}^{o}[k]
\end{aligned}
$$

satisfy the reconstruction condition ([6). Here $s[m]$ are free parameters.

To compute the dyadic wavelet transform and its inverse, the following proposition is very useful.

Proposition 2 (Ref.[4]). Under the condition (6), the relations

$$
\begin{array}{ll}
a_{j+1}[n]=\sum_{k} h[k] a_{j}\left[n+2^{j} k\right], & j=0,1, \ldots, \\
d_{j+1}[n]=\sum_{k} g[k] a_{j}\left[n+2^{j} k\right], & j=0,1, \ldots,
\end{array}
$$


and

$$
a_{j}[n]=\frac{1}{2} \sum_{k}\left(\tilde{h}[k] a_{j+1}\left[n-2^{j} k\right]+\tilde{g}[k] d_{j+1}\left[n-2^{j} k\right]\right), \quad j=0,1, \ldots
$$

hold. Here $a_{0}[n]$ is given by $a_{0}[n]=\int_{-\infty}^{\infty} f(t) \phi(t-n) d t$.

In the case of images, these formulas are applied in each direction, that is, the horizontal and vertical direction. To describe more precisely, let $C^{j}[n, m]$, $D_{1}^{j}[n, m], D_{2}^{j}[n, m]$, and $D_{3}^{j}[n, m]$ indicate low frequency components, high frequency components in horizontal, in vertical, and in diagonal directions, respectively. The indices $n$ and $m$ are the locations in vertical and horizontal directions, respectively. At first, we apply (8) to $C^{j}[n, m]$ in the vertical direction to construct $D_{1}^{j+1}[n, m]$, and we put

$$
C^{j, r o w}[n, m]=\sum_{k} h[k] C^{j}\left[n, m+2^{j} k\right] .
$$

Then, applying (9) to $C^{j, r o w}[n, m]$ in the horizontal direction, we obtain

$$
D_{1}^{j+1}[n, m]=\sum_{l} g[l] C^{j, r o w}\left[n+2^{j} l, m\right] .
$$

Similarly, applying (8) to $C^{j}[n, m]$ in the horizontal direction, and we set

$$
C^{j, c o l}[n, m]=\sum_{k} h[k] C^{j}\left[n+2^{j} k, m\right] .
$$

Using this relation and (9), we get

$$
D_{2}^{j+1}[n, m]=\sum_{l} g[l] C^{j, c o l}\left[n, m+2^{j} l\right] .
$$

Applying (9) twice in each direction, we can obtain $D_{3}^{j}[n, m]$.

\section{$3 \quad$ Learning Method}

In this section, we describe how to determine free parameters $s[m]$ in (7). To distinguish free parameter of the filters in vertical and horizontal direction, we use the symbols $g_{d}[k]$ and $g_{e}[k]$ corresponding to vertical and horizontal directions, respectively. Then, by Proposition 1, we can obtain

$$
\begin{aligned}
& g_{d}[k]=g^{o}[k]-\sum_{m} s_{d}[m] h^{o}[k-m] \\
& g_{e}[k]=g^{o}[k]-\sum_{m} s_{e}[m] h^{o}[k-m],
\end{aligned}
$$

where $s_{d}$ and $s_{e}$ are free parameters. 
Substituting (13) and (14) for (11) and (12), we obtain the following relations.

$$
\begin{aligned}
D_{1}^{j+1}[n, m] & =\sum_{k} g_{d}[k] C^{j, c o l}\left[n, m+2^{j} k\right] \\
& =\sum_{k} g^{o}[k] C^{j, c o l}\left[n, m+2^{j} k\right]-\sum_{l} s_{d}[l] \sum_{k} h^{o}[k-l] C^{j, c o l}\left[n, m+2^{j} k\right] \\
& =\hat{D}_{1}^{j+1}[n, m]-\sum_{l} s_{d}[l] \hat{C}^{j+1}[n, m] \\
D_{2}^{j+1}[n, m] & =\sum_{k} g_{e}[k] C^{j, r o w}\left[n+2^{j} k, m\right] \\
& =\sum_{k} g^{o}[k] C^{j, r o w}\left[n+2^{j} k, m\right]-\sum_{l} s_{e}[l] \sum_{k} h^{o}[k-l] C^{j, r o w}\left[n+2^{j} k, m\right] \\
& =\hat{D}_{2}^{j+1}[n, m]-\sum_{l} s_{e}[l] \hat{C}^{j+1}[n, m],
\end{aligned}
$$

where we put $\hat{D}_{1}^{j+1}[n, m]=g^{o}[k] C^{j, c o l}\left[n, m+2^{j} k\right] \quad \hat{D}_{2}^{j+1}[n, m]=$ $\sum_{k} g^{o}[k] C^{j, \text { row }}\left[n+2^{j} k, m\right]$ and $\hat{C}^{j+1}[n, m]=C^{j, \text { row }}\left[n+2^{j} k, m\right]=C^{j, \text { col }}[n, m+$ $\left.2^{j} k\right]$.

To simplify the onward discussion, we discuss only wavelet decomposition from level 0 to 1 . Let $C^{0}[n, m]$ be an noisy image, then $\hat{D}_{1}^{1}[n, m]$ and $\hat{D}_{2}^{1}[n, m]$ are high frequency components of $C^{0}[n, m]$. Since the high frequency components of the noisy image contain the information of noise in general, we determine free parameters $s_{d}$ and $s_{e}$ so as to vanish the high frequency components $D_{1}^{1}[n, m]$ and $D_{2}^{1}[n, m]$ as follows:

$$
\begin{aligned}
& D_{1}^{1}[n, m]=\hat{D}_{1}^{1}[n, m]-\sum_{l^{\prime}} s_{d}\left[l^{\prime}\right] \hat{C}^{1}[n, m]=0 \\
& D_{2}^{1}[n, m]=\hat{D}_{2}^{1}[n, m]-\sum_{k^{\prime}} s_{e}\left[k^{\prime}\right] \hat{C}^{1}[n, m]=0 .
\end{aligned}
$$

Since $D_{1}^{1}[n, m]$ and $D_{2}^{1}[n, m]$ contain several free parameters $s_{d}$ and $s_{e}$, we prepare $2 N$ exactly similar images $\hat{C}^{1, \nu}(\mu=1,2, \ldots, 2 N)$ as training patterns ,and impose on them the following conditions:

$$
\begin{gathered}
\hat{D}_{1}^{1, \nu}[n, m]-\sum_{l^{\prime}=m-N}^{m+N} s_{d}\left[l^{\prime}\right] \hat{C}^{1, \nu}[n, m]=0 \quad \nu=1,2, \cdots, 2 N \\
\hat{D}_{2}^{1, \nu}[n, m]-\sum_{k^{\prime}=n-N}^{n+N} s_{e}\left[k^{\prime}\right] \hat{C}^{1, \nu}[n, m]=0 \quad \nu=1,2, \cdots, 2 N,
\end{gathered}
$$

where $\hat{D}_{1}^{1, \nu}[n, m]$ and $\hat{D}_{2}^{1, \nu}[n, m]$ are the frequency components $\hat{C}^{1, \nu}[n, m]$ is the low frequency component based on the initial initial filters.

The number of $s_{d}$ and $s_{e}$ is $2 N+1$, but the number of equations (15) and (16) is $2 N$, respectively. We need one more condition for each of $s_{d}$ and $s_{e}$ 
to determine uniquely. Since $g_{d}$ and $g_{e}$ are highpass filters, these filters should satisfy

$$
\begin{aligned}
& \sum_{k} g_{d}[k]=\sum_{k}\left(g^{o}[k]-\sum_{l^{\prime}=m-N}^{m+N} s_{d}\left[l^{\prime}\right] h^{o}\left[k-l^{\prime}\right]\right)=0, \\
& \sum_{k} g_{e}[k]=\sum_{k}\left(g^{o}[k]-\sum_{k^{\prime}=n-N}^{n+N} s_{e}\left[k^{\prime}\right] h^{o}\left[k-k^{\prime}\right]\right)=0 .
\end{aligned}
$$

Therefore

$$
\sum_{l^{\prime}=m-N}^{m+N} s_{d}\left[l^{\prime}\right]=0 \text { and } \sum_{k^{\prime}=n-N}^{n+N} s_{e}\left[k^{\prime}\right]=0
$$

hold, because $\sum_{k} g^{o}[k]=0$ and $\sum_{k} h^{o}[k]$ is constant. Then we can obtain $s_{d}$ by solving the linear equation (15) and (17) using a certain numerical method, for example, the Gaussian elimination method. Similarly, solving the linear equation (16) and (17), we can get $s_{e}$.

\section{Edge Preserving Wavelet Thresholding}

In general, denoising algorithms remove not only noise but also important image features. To improve the the image quality as possible, we employ the modified version of the edge-preserving wavelet thresholding proposed in [8] for preserving image features.

Let $\bar{C}[n, m]=C[n, m]+E[n, m]$ be a noisy image , and $C[n, m]$ and $E[n, m]$ correspond to the original image and noise, respectively. We consider the following minimization problems which find $\dot{D}_{r}^{1}$ as the minimizer of a penalized least-square functionals $F_{p}\left(D_{r}^{1}\right)$.

$$
\dot{D}_{r}^{1}=\operatorname{argmin} F_{p}\left(D_{r}^{1}\right)=\operatorname{argmin}\left(\left\|\bar{D}_{r}^{1}-D_{r}^{1}\right\|_{2}^{2}+\lambda p\left(D_{r}^{1}\right)\right), \quad r=1,2,3,
$$

where $p\left(D_{r}^{1}\right)$ is a given penalty function and $\lambda$ is a positive parameter which balances the effect of the data fidelity and the penalization terms, $\bar{D}_{r}^{1}$ and $D_{r}^{1}$ are the high frequency components in each direction corresponding to $\bar{C}$ and $C$, respectively.

We represent the strength of edges as

$$
M[n, m]=\sqrt{\left|\left(D_{1}^{1}[n, m]\right)\right|^{2}+\left|\left(D_{2}^{1}[n, m]\right)\right|^{2}+\left|\left(D_{3}^{1}[n, m]\right)\right|^{2}},
$$

and use as penalty term the following expression:

$$
p(D)=\sum_{n, m} \varphi(M[n, m])
$$

to preserve the value of $M[n, m]$ as possible. Here $\varphi$ is chosen to be one of the most used potential function of nonlinear diffusion filtering method. In our method, we set 


$$
\varphi(M[n, m])=\mu\left(\sqrt{\mu^{2}+M[n, m]^{2}}-\mu\right)
$$

which is one of functions presented in [8], and the parameter $\mu$ plays the role of a scale-dependent contrast parameter representing the threshold between edge and not-an-edge. We set

$$
\mu=1.4826 \cdot\left(\sum_{r=1}^{3} \operatorname{MAD}\left[\left(\bar{D}_{r}[n, m]\right)^{2}\right]_{n, m=1}^{N^{2}}\right) .
$$

This value comes from the classical tools in the robust statistics, and MAD denotes the median absolute deviation. Then, according to 8], the denoising algorithm is reduced to the solving of non-linear equations

$$
\dot{D}_{r}^{1}[n, m]+\frac{\lambda}{2} \varphi^{\prime}(\dot{M}[n, m])=\left|\bar{D}_{r}^{1}[n, m]\right| \quad r=1,2,3
$$

Using a certain iterative approach, we can obtain approximate solutions of (19).

\section{Denoising Algorithm}

We describe the our denoising algorithm based on the arguments in previous sections.

Since the number of $s_{d}$ and $s_{e}$ is equal to the one of $h[k]$ and $g[k]$, we have to decompose the original image into sub-images depending on the number of these parameters, and then apply the dyadic lifting schemes to each sub-images. If the support length is $\alpha$, the size of each sub-images is $\alpha \times \alpha$. Let us denote the each sub-images as $C_{i}^{0}(1 \leq i \leq M)$, and we decompose $C_{i}^{0}(1 \leq i \leq M)$ into the frequency components $C_{i}^{1}[n, m], D_{1, i}^{1}[n, m], D_{2, i}^{1}[n, m]$, and $\bar{D}_{3, i}^{1}[n, m]$ by using $g_{d}^{i}[k]$ and $g_{e}^{i}[k]$ described in Sections 3. Then, we solve the equation (19) at each sub-image using iterative method as follows:

1. Set the initial values $\left(D_{1}\right)_{0},\left(D_{2}\right)_{0},\left(D_{3}\right)_{0}$ and $\tau=0$. Here $\tau$ stands for the iteration number.

2. Compute $M_{\tau}=\sqrt{\left(D_{1}\right)_{\tau}^{2}+\left(D_{2}\right)_{\tau}^{2}+\left(D_{3}\right)_{\tau}^{2}}$ and

$$
\begin{aligned}
y_{\tau+1}^{r} & =\varphi^{\prime}\left(M_{\tau}\right) /\left(2\left(D_{r}\right)_{\tau}\right) \\
\left(D_{r}\right)_{\tau+1} & =\left|D_{r, i}^{1}\right| /\left(1+\lambda y_{\tau+1}^{r}\right), \quad r=1,2,3 .
\end{aligned}
$$

3. If $\left(D_{r}\right)_{\tau}$ converge when $\tau=\hat{\tau}$, then stop and move to the next step. If not, set $\tau \leftarrow \tau+1$ and return to step 2 .

4. Using $\left(D_{r}\right)_{\hat{\tau}}$, compute

$$
\dot{D}_{r}^{1}[n, m]=\left(D_{r}\right)_{\hat{\tau}}[n, m] \cdot \operatorname{sign}\left(D_{r}^{1}[n, m]\right), \quad r=1,2,3 .
$$

5. Using $C^{1}[n, m], \dot{D}_{1}^{1}[n, m], \dot{D}_{2}^{1}[n, m], \dot{D}_{3}^{1}[n, m]$ and the initial filters, reconstruct the image.

Fig 1 shows our denoising process. 


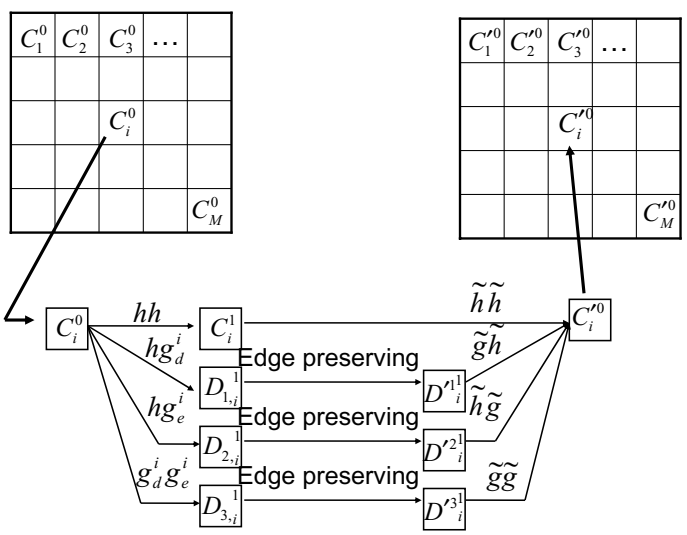

Fig. 1. Denoising algorithm

\section{$6 \quad$ Numerical Results}

We employ the well-known Lenna, Boat and Title grayscale images each of size $256 \times 256$ as benchmarks, shown in Fig 3 . The noisy images corrupted by adding white Gaussian noise are shown in Fig 4 . We have used the $64 \times 64$ pixel fragment of "Title" to highlight the edge preservation properties in Fig 8 . The performance of the estimators was measured by the usual peak signal to ratio(PSNR). In this simulation, we employ the spline dyadic wavelet filters as initial filters shown in Table 1

Table 1. Initial filter

\begin{tabular}{|c||c|c|c|c|}
\hline $\mathrm{n}$ & $h^{o}[n] / \sqrt{2}$ & $g^{o}[n] / \sqrt{2}$ & $\tilde{h}^{o}[n] / \sqrt{2}$ & $\tilde{g}^{o}[n] / \sqrt{2}$ \\
\hline-2 & & & & -0.03125 \\
\hline-1 & 0.125 & & 0.125 & -0.21875 \\
\hline 0 & 0.375 & -0.5 & 0.375 & -0.6875 \\
\hline 1 & 0.375 & 0.5 & 0.375 & 0.6875 \\
\hline 2 & 0.125 & & 0.125 & 0.21875 \\
\hline 3 & & & & 0.03125 \\
\hline
\end{tabular}

Table 2 lists the value of PSNR for various version of the benchmarks recovered from their noisy versions using median filter and our method. In Proposed method 1, we determine the free parameters using the method described in section 3. On the other hand, we determine the free parameters so as to preserve the features of initial filters and adjust the peak based on our experiences. The shape of the initial filter and the determined filter in Proposed method 2 is showed in Fig,2.

Therefore Proposed method 2 is not theoretical but intuitive, however, Table 2 shows that Proposed method 2 is superior to Proposed method 1 in 


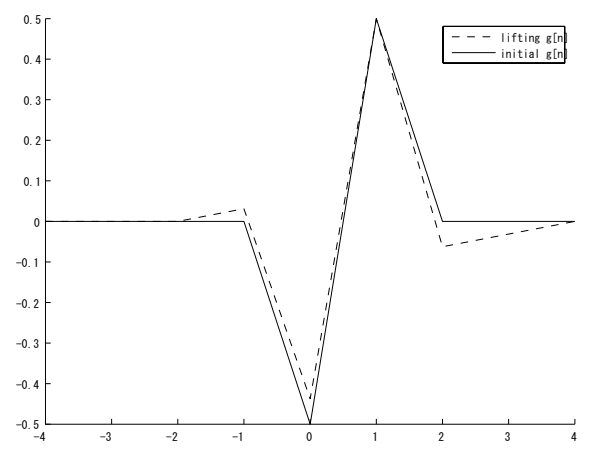

Fig. 2.

Table 2. PSNR

\begin{tabular}{l|c||c|c|c|c}
\hline & & Noisy & Median & Proposed method1 & Proposed method2 \\
\hline \multirow{4}{*}{ PSNR } & \multirow{3}{*}{ Lenna } & 16.1335 & 22.5340 & 23.1725 & 25.0105 \\
& & 18.0880 & 24.1028 & 24.6104 & 26.0055 \\
& \multirow{2}{*}{ Boat } & 20.0476 & 25.6968 & 25.8689 & 26.7741 \\
\cline { 3 - 6 } & 16.0252 & 22.5085 & 23.2077 & 25.2972 \\
& & 18.0034 & 24.1800 & 24.8185 & 26.4044 \\
& Title & 11.7421 & 12.9030 & 14.0684 & 27.1942 \\
\hline
\end{tabular}

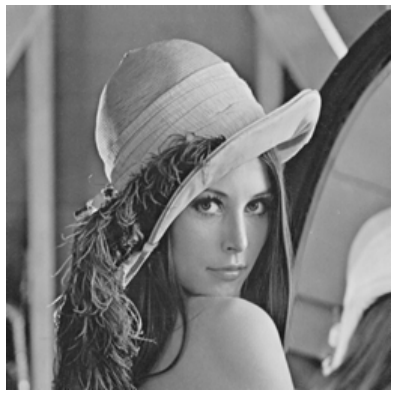

(a)

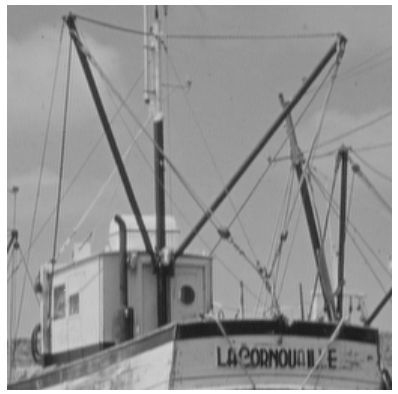

(b)

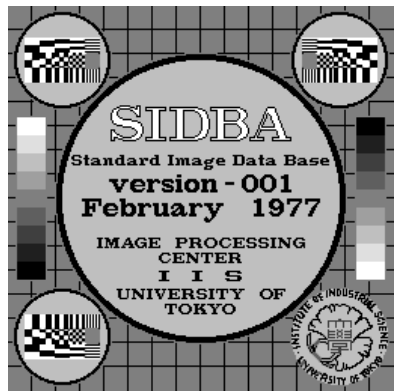

(c)

Fig. 3. (a) Lenna image. (b) Boat image. (c) Title image.

many cases. Reversely, Figs 5 demonstrate that Proposed method 1 preserves the edges in comparison with other methods. This means that the choice of the free parameters is very important for denoising. Anyway, both Proposed method 1 and 2 are superior to median filter. 


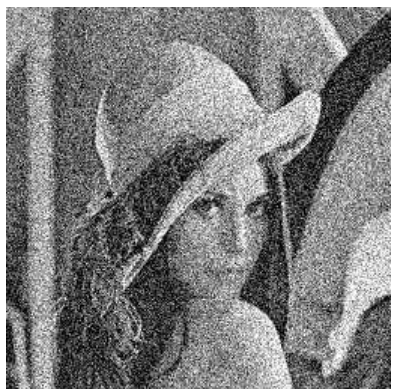

(a)

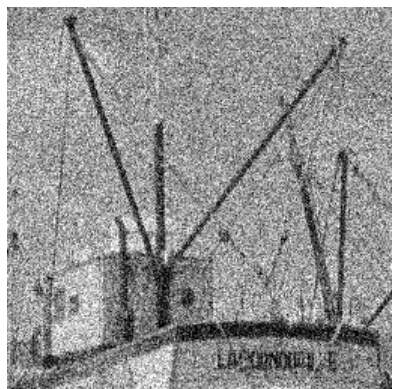

(b)

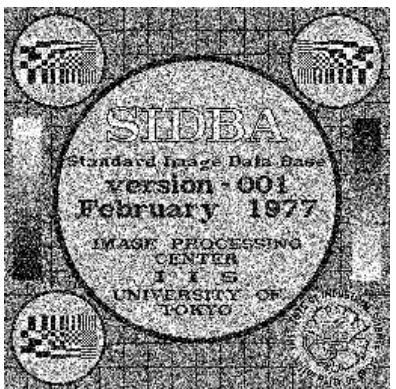

(c)

Fig. 4. (a) Noisy Lenna(PSNR=16.1355).

Noisy Title $(\mathrm{PSNR}=11.7421)$.

(b) Noisy Boat(PSNR $=16.0252)$.

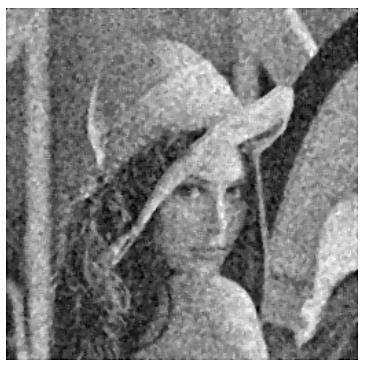

(a)

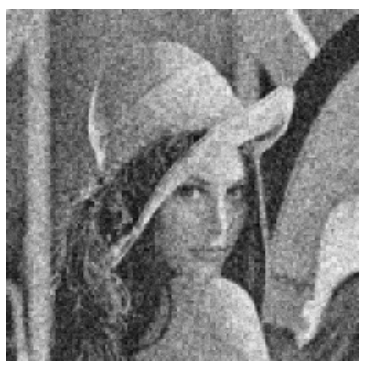

(b)

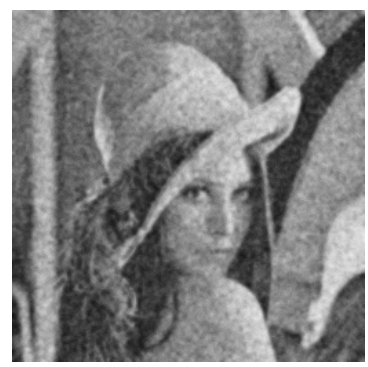

(c)

Fig. 5. (a) Median(PSNR $=22.5340)$. Proposed method2(PSNR $=25.0105)$.

(b) Proposed method1(PSNR $=23.1725)$.

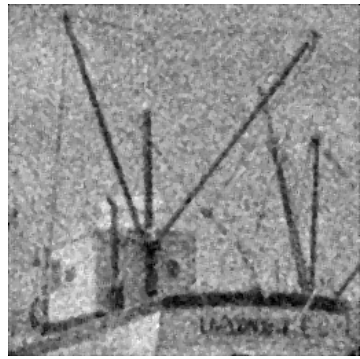

(a)

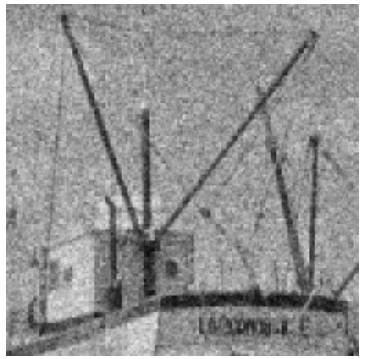

(b)

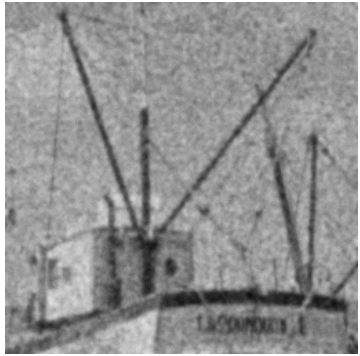

(c)

Fig. 6. (a) Median(PSNR $=22.5085)$. Proposed method2(PSNR $=25.2972)$.

(b) Proposed method1(PSNR= 23.2077). 


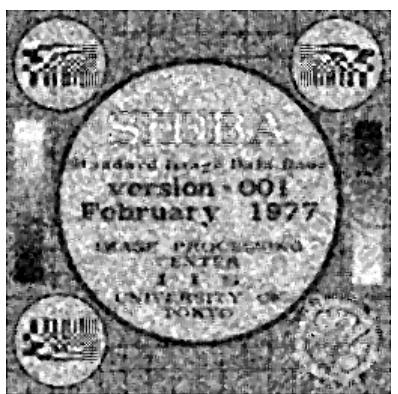

(a)

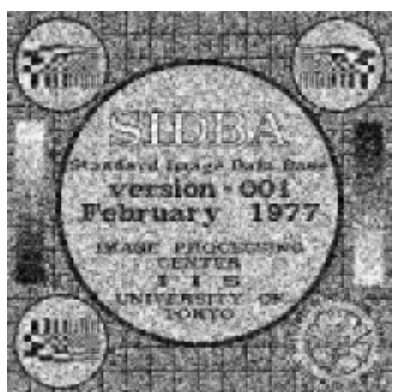

(b)

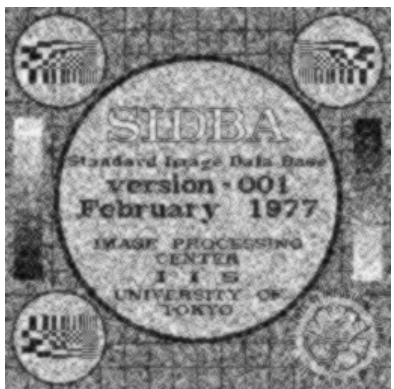

(c)

Fig. 7. (a) Median(PSNR $=12.9030)$.

Proposed method2(PSNR $=14.0713)$.

(b) Proposed method1(PSNR $=14.0684)$.

(c)

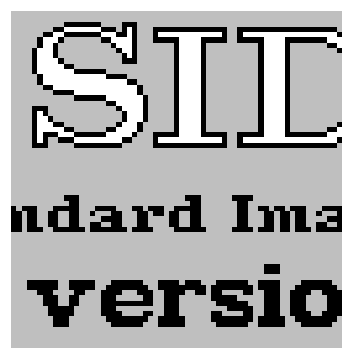

(a)

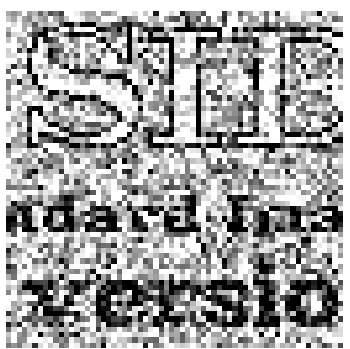

(b)

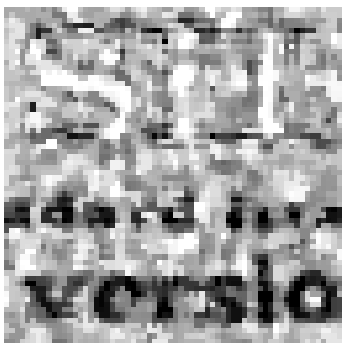

(c)

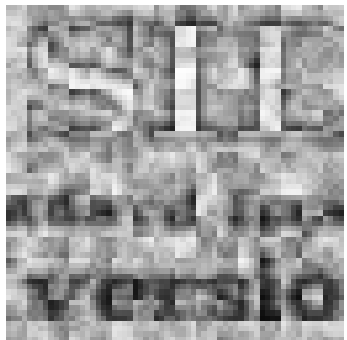

(d)

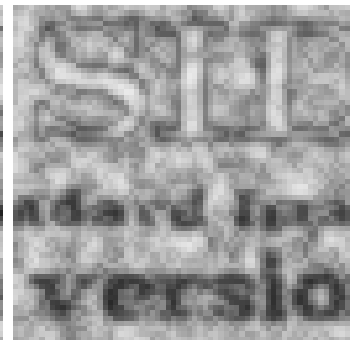

(e)

Fig. 8. (a) Original image.

(b) Noisy image.

(c) Median.

(d) Proposed method1.

\section{Conclusion}

We proposed a new wavelet denoising method for digital images with edge preservation. Once we give the initial filter, our denoising method is carried out automatically thanks to the learning method. The performance of our method is compared to that of median filters in numerical experiments. Our numerical 
results show that our methods work well in comparison with median filter. Since the free parameters appearing in the dyadic lifting schemes play an important role to denoise, the development of new learning methods to determine the best parameters remains as a future work.

\section{References}

1. Aubert, G., Kornprobst, P.: Mathematical problems in image processing, 2nd edn. Partial differential equations and the calculus of variations. Springer, Heidelberg (2006)

2. Donoho, D.L.: De-noising by soft-thresholding. IEEE Trans. Inform. Theory 41(3), 613-627 (1995)

3. Mallat, S., Zhong, S.: Characterization of signals from multiscale edges. IEEE trans. pattern anal. mach. intell. 14(7), 710-732 (1992)

4. Mallat, S.: A wavelet tour of signal processing. Academic press, London (1998)

5. Papari, G., Campisi, P., Petkov, N., Neri, A.: Contour detection by multiresolution surround inhibition. In: Proc. Int. Conf. on Image Processing ICIP 2006, Atlanta, GA, October 8-11, pp. 749-752 (2006)

6. Türüki, T.A., Hussain, M., Niijima, K., Takano, S.: The dyadic lifting schemes and the denoising of digital images. International Journal of Wavelets, Multiresolution and Information Processing 6(3), 331-351 (2008)

7. Sweldens, W.: The lifting scheme:A construction of second generation wavelets. SIAM J. Math. Anal. 29(2), 511-546 (1997)

8. Lazzaro, D., Montefusco, L.B.: Edge-preserving wavelet thresholding for image denoising. J. Comput. Appl. Math. 210, 222-231 (2007) 